\title{
Sublimation of organic-rich comet analog materials and their relevance in fracture formation ${ }^{\star}$
}

\author{
David Haack ${ }^{1}$, Christopher Kreuzig ${ }^{2}$, Bastian Gundlach ${ }^{2}$, Jürgen Blum² ${ }^{2}$ and Katharina Otto ${ }^{1}$ \\ ${ }^{1}$ Institut für Planetenforschung, Deutsches Zentrum für Luft- und Raumfahrt (DLR), Berlin, Germany \\ e-mail: david.haack@dlr.de \\ ${ }^{2}$ Institut für Geophysik und Extraterrestrische Physik, Technische Universität Braunschweig, Braunschweig, Germany
}

Received 23 August 2021 / Accepted 6 September 2021

\begin{abstract}
Aims. The morphology of cometary nuclei is the result of an ongoing evolution and can provide valuable information to constrain the composition of comets. In our laboratory experiments we investigated the morphological evolution of comet analog materials, which consist of volatile, dust, and organic components. The laboratory results are aimed to help understand the evolution of cometary surfaces.

Methods. We used spherical particles of fly ash and mixtures of ice, glycine, and sodium acetate as analog materials in different mass ratios to reproduce observed cometary morphologies. The cohesive and gravitational properties in the laboratory are scaled to cometary conditions to draw meaningful conclusions from the experimental results. The samples were placed in a vacuum sublimation chamber, cooled down to below $150 \mathrm{~K}$, and were insolated with an external light source. To analyze the morphology of the samples, a camera was used to monitor the alterations of the surface.

Results. Organic components in volatile-rich samples can have a distinct adhesive effect after the volatiles sublimate. During the sublimation process the sample volume decreases and fractures form on the sample surface. Due to the stability of the remaining volatile-depleted material, significant cliff collapses or ejected particles were not observed in the laboratory.
\end{abstract}

Key words. comets: general - methods: laboratory: solid state - planets and satellites: formation

\section{Introduction}

Comets are primordial objects from the early days of the Solar System. Due to their origin from the outer Solar System beyond the ice lines of volatile materials (Dodson-Robinson et al. 2009), they have been subject to no or only minor alterations since their formation and thus are archives with information about the early Solar System. This makes them interesting targets for space missions that have studied comets at close range since the mid-1980s (e.g., ESA's Giotto and Rosetta missions and NASA's Stardust and Deep Impact missions). Even with these missions, the processes leading to the formation of comets and their evolution are not yet fully understood (Weissman et al. 2020). Which amounts of volatile and nonvolatile materials are present in a cometary nucleus and which processes alter a comet's surface as it approaches the Sun are still the subject of investigation (Gulkis et al. 2015; Sierks et al. 2015). The sublimation of volatile components, such as $\mathrm{CO}, \mathrm{CO}_{2}$, or $\mathrm{H}_{2} \mathrm{O}$ (Hässig et al. 2015; Läuter et al. 2018; Biver et al. 2019), is caused by the insolation of solar energy to the surface and the heat transfer to the shallow subsurface of a comet in an airless environment. During this process, layers of nonvolatile mineralic and organic components (Capaccioni et al. 2015; Goesmann et al. 2015; Raponi et al. 2020) accumulate at the surface and can be redeposited or transported away by the gas drag of continuously sublimating volatiles (Kührt \& Keller 1994; Gundlach et al. 2015, 2020; Keller et al. 2017; Bischoff et al. 2019). With each orbit around the Sun, a comet loses more material via progressive erosion

\footnotetext{
${ }^{\star}$ Movies are available at https://www . aanda.org
}

(Thomas et al. 2015a) and a variety of morphologies are formed on its surface, such as cliffs, fractures, and rocky or flat areas (Thomas et al. 2015b; Pajola et al. 2016; El-Maarry et al. 2019). The appearance of morphological features are determined by the chemical composition, grain size, porosity, and cohesive and gravitational forces of the comet's nucleus. Therefore, the reproduction and analysis of cometary morphologies in the laboratory may provide information about the composition and evolutionary history of comets in space.

Laboratory experiments, such as the KOSI (KOmetenSImulation) project in the $1990 \mathrm{~s}$, have studied sublimation processes that are expected to take place on comets (Grün et al. 1989, 1993; Lämmerzahl et al. 1995). As part of the KOSI project, a sample container was filled with mixtures of nonvolatile ana$\log$ materials and ices and was placed in a sublimation chamber. After the samples were insolated with an energy source, an ice-free layer formed on their surfaces, preventing energy transport to underlying layers and further sublimation processes after a few hours. With the Rosetta mission and its landing module Philae, cometary science and laboratory analog experiments increasingly came into focus. The measurements and detailed images of comet 67P/Churyumov-Gerasimenko (hereafter 67P) provided by the spacecraft significantly increased our knowledge of comets. Nevertheless, the influence of detected volatiles, organic compounds, and their abundances (e.g., Hässig et al. 2015; Läuter et al. 2018; Capaccioni et al. 2015; Raponi et al. 2020) on the evolution of the comet remains a research objective vital to the interpretation of cometary surfaces.

In order to more precisely determine the properties of materials that could be considered for analog experiments, several 
series of measurements have been performed. It was found that the tensile strength of water ice at temperatures below $150 \mathrm{~K}$ is much lower than expected (Gundlach et al. 2018b; Musiolik \& Wurm 2019). This temperature limit should not be exceeded in the experiment; if so, water ice particles start to sinter and stiff connections form between the particles (Kuroiwa 1961; Gundlach et al. 2018a). Furthermore, it was shown that spherical particles could produce samples whose low tensile strengths and filling factors are closer to those of comets than of samples made of angular particles (Haack et al. 2020). The filling factor describes the ratio of a volume filled with particles. Besides applications in the laboratory, these findings were also used to calibrate numerical models of comet simulations (Kappel et al. 2020).

Analog experiments with the Simulation Chamber for Imaging the Temporal Evolution of Analogue Samples (SCITEAS; Pommerol et al. 2015) allowed photometric studies and visual observation of sublimating sample surfaces at temperatures and pressures similar to those expected on the surface of comets at low heliocentric distances. Poch et al. (2016a,b) used SCITEAS to investigate the influence of organic and mineralic components on the sublimation behavior of water ice. They found that the texture of the residuals after sublimation of the ice differed significantly depending on whether the nonvolatile components were included in the ice matrix (intra-mixtures) or were added as pure substances to pure ice particles (inter-mixture). The residuals of intra-mixtures were extremely porous and foam-like, and cohesive large flakes sometimes ejected from the surface. Inter-mixtures formed more compact and less cohesive residuals on the sample surface during the sublimation process. However, the aim of these experiments was not to realistically reproduce cometary morphologies. Therefore, the amount of nonvolatile materials accounted for less than one percent by mass in the mixtures and was not representative of cometary regolith. Kaufmann \& Hagermann (2018) conducted sublimation experiments with water ice and carbon black and, like Poch et al. (2016b), observed a solidified subsurface layer under an icedepleted and low-density cover layer. This suggests the subsurface hardening of volatile-rich and porous materials due to sublimation and recondensation (Kochan et al. 1989; Kossacki et al. 1997).

Most of these experiments were not aimed at reproducing cometary morphologies and were performed with volatile contents of $>90 \mathrm{vol} \%$, which are atypical for comets. To produce more realistic morphologies in the laboratory, Haack et al. (2021) performed sublimation experiments with more representative dust-ice ratios. They used fly ash (a by-product of industrial coal combustion) as the mineralic dust component and water ice particles as the volatile. The amount of ice in the sample material ranged between $66 \mathrm{vol} \%$ and $25 \mathrm{vol} \%$, as presumed for comets (Herique et al. 2017; Fulle et al. 2018; Pätzold et al. 2018). In addition, they allowed horizontal and vertical mass transport of the sample material and found that the resulting sublimation activities and morphologies depend on the insolation angle in addition to the volatile content.

We decided to expand on previous experiments with refractory fly ash and volatile water ice (Haack et al. 2020, 2021) and added organic components as analog materials to the mixtures. The organic components consisted of sodium acetate and glycine in equal parts per mass, which are confirmed on 67P and are nonvolatile materials under our experimental conditions. Based on the findings of the Rosetta satellite mission and laboratory measurements, we prepared sample mixtures of volatile and nonvolatile materials in different ratios as plausible on a comet (Choukroun et al. 2020; Haack et al. 2021). For this, we took advantage of the low tensile strength of granular matter composed of spherical particles at temperatures below $150 \mathrm{~K}$ (Gundlach et al. 2018b; Haack et al. 2020). The aim of the experiments is to investigate the influence of organic components on the sublimation activity and the evolution of the sample morphology and to analyze the results for their relevance with respect to cometary surfaces.

\section{Methods}

\subsection{Selection of materials}

As in previous sublimation experiments (Haack et al. 2021), polydisperse fly ash spheres, with an average radius of about $2.4 \mu \mathrm{m}$ and a density of $2.25 \mathrm{~g} \mathrm{~cm}^{-3}$, were used as a refractory dust analog. This material is a compromise between physical and chemical accuracy of dust on a comet. Fly ash corresponds to the mineralic composition of plagioclase (Haack et al. 2021) and is therefore more representative of a mineralic component of a comet compared to particles of pure $\mathrm{SiO}_{2}$. Due to their spherical shape, mutual particle interlocking is prevented. Furthermore, it is possible to produce samples with a very low tensile strength in the laboratory (Haack et al. 2020; Kappel et al. 2020) to simulate the physical properties of cometary materials (Groussin et al. 2015; Attree et al. 2018a). The minerals olivine and pyroxene would be more accurate chemical analogs, but micrometer-sized particles of these minerals are only available with an angular shape. This would result in a drastic increase in the tensile strength of the sample material (Haack et al. 2020) and could reduce or prevent the alteration of the sample surface during the experiments.

As volatile analog material, we used polydisperse water ice spheres with an average radius of about $2.4 \mu \mathrm{m}$. Water ice dominates the volatile materials on 67P (Hässig et al. 2015) and was used as a first grade approximation of the volatile component of a comet. Other volatiles, such as $\mathrm{CO}$ or $\mathrm{CO}_{2}$, contribute 15 mass $\%$ to the amount of volatile materials on 67P (Bockelée-Morvan et al. 2016; Läuter et al. 2018; Biver et al. 2019) and may play an important role in the evolution of a comet but were omitted to limit the complexity of the experiments. This is a reasonable limitation because in our experiments we simulated cometary conditions in which the sublimation of water may predominate since $\mathrm{CO}$ or $\mathrm{CO}_{2}$ are more volatile and would already be sublimated in the simulated scenario. However, the influence of other ices on the activity of a simulated comet should be studied in future experiments.

The additional third component should be a combination of organic materials that is nonvolatile both on a cometary surface and under experimental conditions. Since the composition of the organic components of a comet is very complex (Capaccioni et al. 2015; Goesmann et al. 2015; Altwegg et al. 2017), we had to limit the number of organic components in our experiments to a few materials that may represent the chemical properties of a large variety of organic materials on a comet. We used a combination of sodium acetate $\left(\mathrm{NaCH}_{3} \mathrm{COO}\right)$ and glycine $\left(\mathrm{C}_{2} \mathrm{H}_{5} \mathrm{NO}_{2}\right)$ in equal amounts, which are confirmed as components of 67P (e.g., Elsila et al. 2009; Altwegg et al. 2016; Rubin et al. 2019; Schuhmann et al. 2019). Sodium acetate represents organic ions and salts, and glycine is a simple amino acid and stands for a group of organic components that can potentially form larger and complex molecules. This selection of organic analog materials is very limited and cannot reflect the complexity of organic materials on a comet; however, this was inevitable since the 
actual composition of organic components on a comet is not fully understood and we had to limit the complexity of the experiments. Other combinations of organic materials could be conceivable, but a limited selection would generally not be able to simulate the complete variety of organic material on a comet. An additional reason for the chemical limitation was the feasibility of the experiments in terms of the components' toxicity. The nonvolatile character of the organic components was confirmed in previous measurements with the same experimental setup.

We omitted the usage of additional materials to reduce the sample's albedo. This is not realistic because comets have a very low albedo (Keller et al. 1986; Ciarniello et al. 2015) and absorb insolated radiation much better than the analog material. However, this effect was taken into account in the energy scaling (see Haack et al. 2021) and led to increased insolation rates in the laboratory. This allowed the direct comparison of the obtained results with previous sublimation experiments without organic components (Haack et al. 2021), where all samples had an albedo of $\sim 0.35$. This decision also kept the complexity of the experiment within limits and reduced the number of possibly interfering effects at the sample's surfaces.

\subsection{Sample preparation}

To keep the results comparable to those of previous experiments, the sample preparation method was adapted from Gundlach et al. (2018b) and Haack et al. (2020). Water mist was generated with a droplet dispenser and sprayed into a Dewar vessel containing liquid nitrogen. The droplets froze in the liquid nitrogen to form polydisperse spherical ice particles and sank to the bottom of the container.

We modified this method: the droplet dispenser was filled with an aqueous solution of the organic molecules instead of pure water. For this, sodium acetate and glycine were mixed in equal mass ratios (hereafter referred to as organics) and completely dissolved in water. In order to study the influence of different ratios of organic materials and water, two solutions were prepared, with the organic-to-water mass ratios of 10:100 and 5:100.

The resulting particles in the Dewar vessel consisted of organic components enclosed in an ice matrix. The advantage of this method was that the pure organic analog materials did not have to be shaped into micrometer-sized spheres to be comparable to fly ash and water ice particles. In addition, Hadraoui et al. (2019) suggested that glycine embedded in a sublimating ice matrix reproduces the emitted glycine detected in the coma of $67 \mathrm{P}$ most realistically.

Archimedes' principle was used to determine the amount of organic-bearing ice particles in the Dewar vessel. The weights of the same volumes of pure liquid nitrogen and of the liquid nitrogen-ice suspension were measured. Using the weight difference of the two measurements and the known densities of liquid nitrogen and ice (Table 1), the mass of ice in the Dewar vessel was calculated. After the amount of ice particles in the vessel was determined, a scale was used to add predefined amounts of fly ash to the suspension. The relative amounts of ice to fly ash were selected between 20 and 40 mass \%; they correspond to the ratios estimated for the nucleus of 67P (Herique et al. 2017; Fulle et al. 2018; Pätzold et al. 2018) and are comparable to those of the sublimation experiments carried out by Haack et al. (2021).

A cylindrical metal mold was filled with the suspensions of organic-bearing ice, fly ash, and liquid nitrogen in the Dewar vessel, which were thoroughly mixed. The mold had an inner diameter of $25 \mathrm{~mm}$ and was placed vertically on a metal sample
Table 1. Molar mass and density of components used to simulate cometary materials.

\begin{tabular}{ccc}
\hline \hline & Molar mass $\left(\mathrm{g} \mathrm{mol}^{-1}\right)$ & Density $\left(\mathrm{g} \mathrm{cm}^{-3}\right)$ \\
\hline Fly ash & & 2.25 \\
$\mathrm{H}_{2} \mathrm{O}$ (ice) & 18.02 & 0.93 \\
$\mathrm{NaCH}_{3} \mathrm{COO}$ & 82.03 & 1.52 \\
$\mathrm{C}_{2} \mathrm{H}_{5} \mathrm{NO}_{2}$ & 75.07 & 1.60 \\
$\mathrm{~N}_{2}$ (liquid) & 28.01 & 0.807 \\
\hline
\end{tabular}

holder (see Haack et al. 2021). The mold and sample holder were cooled with liquid nitrogen before the sample mixture was added in. As the nitrogen of the suspension slowly evaporated, only the ice-organic and fly ash particles remained in the cylinder. When the nitrogen had completely evaporated, the cylindrical mold was removed from the sample holder, uncovering solid but extremely porous samples (Haack et al. 2021) with the diameter of the mold. The samples were immediately placed in the vacuum sublimation chamber prepared for the measurement. This procedure ensured that the sample temperature did not exceed $150 \mathrm{~K}$ and prevented sintering of the ice particles.

To determine the cohesive force between the organic-bearing ice particles, their tensile strength and surface energy were determined with other samples of the same mixture before the sublimation experiments. For this purpose, the Brazilian disk test method was used, as described in previous experiments (Gundlach et al. 2018b; Bischoff et al. 2020; Haack et al. 2020). With a particle radius, $r$, of $2.4 \mu \mathrm{m}$ and a filling factor, $\Phi$, of 0.46 , the tensile strength of ice with 10 mass $\%$ organics at $150 \mathrm{~K}$ amounts to $1300 \pm 250 \mathrm{~Pa}$, compared to $1100 \pm 210 \mathrm{~Pa}$ for pure water ice (Haack et al. 2020). Following Blum et al. (2006), the upper limit for the surface energy, $\gamma$, is

$\gamma=\frac{4 r \sigma}{9 \Phi}$

With the values of the tensile strength, $\sigma$, the surface energy of the used organic-rich ice is $0.0029 \pm 0.0006 \mathrm{~J} \mathrm{~m}^{-2}$, compared to $0.0026 \pm 0.0005 \mathrm{Jm}^{-2}$ for pure water ice (Haack et al. 2020). These values are the same within measurement error, and therefore we can use the previous values of tensile strength and surface energy from Haack et al. (2020) for further calculations of different organic-bearing ice-dust mixtures.

In previous measurements, the albedo of fly ash was determined to be 0.35 . This value was also assumed for our sample mixtures since the albedo of ice-bearing mixtures is mainly determined by the dust component (Chýlek et al. 1983; Clark \& Lucey 1984; Oehler \& Neukum 1991).

\subsection{Scaling to cometary conditions}

To obtain meaningful results from the experiments, the conditions in the laboratory must be scaled to the conditions on a comet. The most important difference between the two environments is the gravity that is by orders of magnitudes higher in the laboratory compared to the $\sim 2 \times 10^{-4} \mathrm{~m} \mathrm{~s}^{-2}$ on comet $67 \mathrm{P}$ (Agarwal et al. 2016). To solve this problem, we used the same scaling for particle size as for the experiments described in Haack et al. (2021). The scaling relates the ratios of gravity and particle-size-dependent cohesion in the laboratory and on 67P. With known gravity $F_{\mathrm{G}}=\frac{4}{3} \pi r^{3} \rho g$ and cohesion $F_{\mathrm{C}}=3 \pi r \gamma$ in the 
laboratory (Haack et al. 2020) and the known gravity on 67P, the corresponding cohesion and thus particle size on the comet can be estimated with

$\frac{F_{\mathrm{G}_{67 \mathrm{P}}}}{F_{\mathrm{C}_{67 \mathrm{P}}}} \approx \frac{F_{\mathrm{G}_{\mathrm{Earth}}}}{F_{\mathrm{C}_{\mathrm{Earth}}}}$.

In these equations, $r$ is the particle radius, $\rho$ is the particle density, $g$ is the gravitational acceleration, and $\gamma$ is the surface energy. According to the determined values of the surface energy and the scaling of Haack et al. (2021), the fly ash and organic-rich ice particles with a radius of $\sim 2.4 \mu \mathrm{m}$ represent millimeter-sized particles on 67P. This is in line with the size of hypothesized pebbles, which may have initially formed comets (Blum et al. 2014, 2017; Fulle et al. 2016; Lorek et al. 2016, 2018). On a macroscopic scale, a $2.5 \mathrm{~cm}$ object in the laboratory would correspond to an object of about $10 \mathrm{~m}$ in size on 67P. This could be a cliff, a large boulder, or other morphological features on the comet (Thomas et al. 2015b; Pajola et al. 2016; El-Maarry et al. 2019) that would be subject to progressive alteration by the sublimation of volatile materials.

After increasing the cohesive forces by reducing the particle size, the insolated energy in the experiment had to be adapted to reflect realistic cometary conditions. The insolated energy heats the ice at the surface, which sublimates and creates a nearsurface vapor pressure according to the Clapeyron-Clausius equation $P_{\mathrm{v}}=A e^{-B / T}$, where $T$ is the temperature of the ice and $A$ and $B$ are constants with values $A=3.56 \times 10^{12} \mathrm{~Pa}$ and $B=6142 \mathrm{~K}$ for water ice (Fanale \& Salvail 1984). The vapor pressure acts on the particle cross section and creates a pressure drag, $F_{\mathrm{D}}=\pi r^{2} P_{\mathrm{v}}$, on the particles of the sample, where $r$ is the particle radius. When the $F_{\mathrm{D}}$ on the particles exceeds their cohesion, $F_{\mathrm{C}}$, individual particles or particle aggregates detach from the sample's surface and can be transported away. To compare this process in the laboratory with $67 \mathrm{P}$, this scaling relates the ratios of insolation-dependent pressure drag and particle cohesion of the sample and on 67P as described in Haack et al. (2021) and follows

$\frac{F_{\mathrm{D}_{67 \mathrm{P}}}}{F_{\mathrm{C}_{67 \mathrm{P}}}} \approx \frac{F_{\mathrm{D}_{\text {Earth }}}}{F_{\mathrm{C}_{\text {Earth }}}}$.

This second scaling ensures that the insolated energy on the sample surface is sufficient to sublimate the water ice and can be related to the corresponding insolation rate on 67P.

When Rosetta reached 67P and monitored activity on its surface, the comet had a heliocentric distance of about $4 \mathrm{AU}$ (Tubiana et al. 2015). According to the energy scaling, this distance can be simulated with $\sim 1$ solar constant in the laboratory. When 67P reaches its perihelion at 1.24 AU, the solar insolation reaches its maximum. This insolation flux would transform to about 33.6 solar constants in the laboratory (Haack et al. 2021). However, the experimental setup was not built to provide this amount of energy. With the solar simulator used in our experiments, a maximum insolation rate of 1.9 solar constants was reachable. This corresponds to a heliocentric distance of 3.6 AU for 67P. Activity has already been observed on the surface of $67 \mathrm{P}$ at this distance (Snodgrass et al. 2013; Tubiana et al. 2015).

\subsection{Sublimation experiments}

The experiments were performed in the vacuum sublimation chamber, which was also used in Haack et al. (2021). Before the sample was placed, the chamber was cooled to about $110 \mathrm{~K}$ and flooded with argon to prevent atmospheric moisture from
Table 2. Mass ratios of the sample mixtures used: relative mass ratios of organics, ice, and dust used for various sample mixtures in the sublimation experiments.

\begin{tabular}{lccc}
\hline \hline & Organics & Ice & Dust (vol\%) \\
\hline Organic free reference & 0 & 100 & $500(67)$ \\
& 5 & 100 & $300(59)$ \\
Low organic content & 5 & 100 & $500(70)$ \\
& 5 & 100 & $700(77)$ \\
High organic content & 10 & 100 & $300(62)$ \\
& 10 & 100 & $500(73)$ \\
\hline
\end{tabular}

Notes. The ice fraction was normalized to 100 for ease of reference. The dust volume with respect to the bulk material is given in parentheses.

entering the chamber when it was opened for sample insertion. The sample holder, with the sample on top, was firmly mounted inside a cooling shield within the chamber and connected to temperature sensors. The cooling shield was a rectangular metal housing, with a side length of $10 \mathrm{~cm}$, which was actively cooled with liquid nitrogen throughout the duration of the experiments. It has two openings, one on its top to allow insolation of the sample from above and one on its side surface for visual observation of the sample (see Fig. 4 in Haack et al. 2021). This mitigated the alteration of the samples by background thermal radiation during the experiments.

After the mounting of the sample holder, the chamber was closed immediately and the pressure and temperature inside were reduced to $\sim 10^{-7}$ mbar and below $150 \mathrm{~K}$, respectively, to simulate conditions similar to those of a comet (Kührt 1984). When pressure and temperature reached operating values, a solar simulator was used to insolate the sample vertically from above with an insolation flux of 1.9 solar constants. According to the energy scaling, this corresponds to a heliocentric distance of about 3.6 AU. To verify the temperature stability during the experiments, temperature sensors were used to monitor the sample and the cooling shield. A camera with a $3000 \times 2000$ pixel sensor was focused at the sample through a side window of the sublimation chamber and captured images $(25 \mu \mathrm{m}$ per pixel $)$ at a frame rate of one image per second.

Two series of experiments with three measurements each were performed with two different fixed organic-ice mass ratios, 5:100 and 10:100. The subsequently added amount of dust led to 300:100, 500:100, and 700:100 mass ratios with respect to ice for both series (Table 2). This represents ice-dust ratios as suggested by observations on 67P (Herique et al. 2017; Fulle et al. 2017, 2018) and by laboratory experiments (Haack et al. 2021). A reference measurement was performed without organics to directly infer the influence of organics on the evolution of the sample morphology. The insolation stopped after a maximum of $20 \mathrm{~h}$ of measurements, when the supply of liquid nitrogen to cool the sublimation chamber had to be replenished. At the end of the experiment, the images taken with the camera were analyzed in terms of the degree of alterations on the sample surface with respect to the sample compositions.

\section{Results}

To obtain a reference measurement of organic-free material, a sample with an ice-dust mass ratio of 100:500 was placed in the 

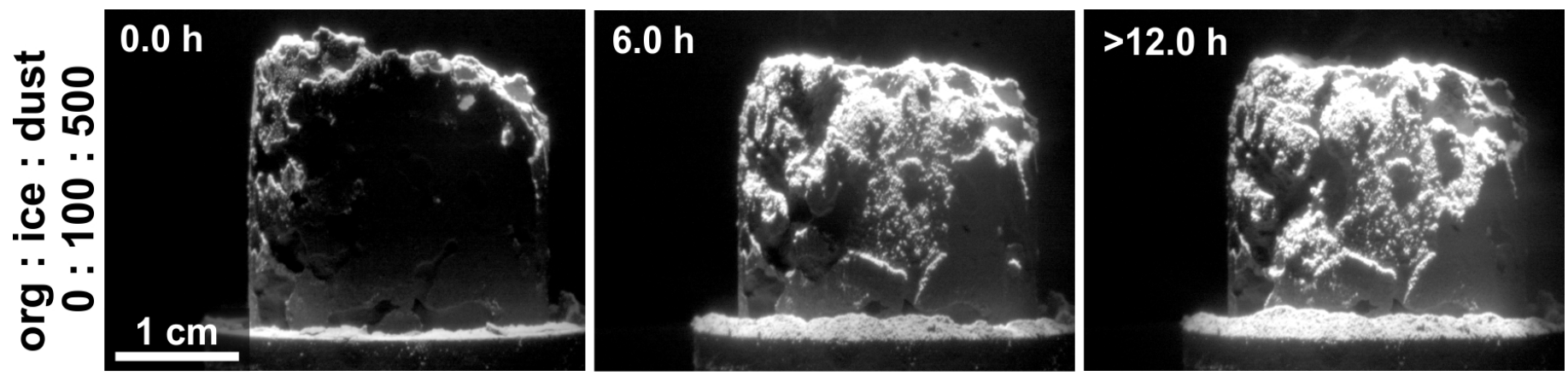

Fig. 1. Sublimation experiment without organic components as reference. It was performed to obtain a reference to samples with organic components. The series shows how the surface of a sample with an ice-dust mass ratio of 100:500 evolves under a vertical insolation of 1.9 solar constants. The shape of the sample altered as material detached and fell off the lateral surfaces as individual grains or local cliff collapses. No further surface alterations were observed after $12 \mathrm{~h}$. A time lapse of the evolution is available as an online movie.

chamber. With vertical insolation, particles were only sporadically ejected from the top in the first minutes of the experiment. Significant surface alteration was limited to the vertical surface. Material detached from the vertical side wall and fell down due to gravity. In this process, larger areas of the wall collapsed repeatedly. This altered the shape of the sample from cylindrical to more conical, with decreasing diameter toward the top (Fig. 1). A difference between the albedo of ice-depleted and fresh material could not be detected.

Areas where fresh material was exposed to the sample surface appeared very rough, with a granular texture in the submillimeter range. The detached material accumulated directly at the base of the sample as ice-depleted granular dust. The surface alteration rate decreased throughout the experiment, and after about $12 \mathrm{~h}$ the activity came to an end. Unfortunately, the experimental setup was not designed to monitor mass loss during the experiments, so the results were limited to visual observations.

By the end of the experiment, an ice-depleted non-cohesive dust layer had formed on the remaining sample material, and large amounts of ice were observed in the center of the remains. The remaining ice-rich material had solidified significantly compared to the sample material at the start of the experiment. However, with the setup used, it was not possible to determine the exact amount of remaining ice and the sample's tensile strength because frost formed on the sample immediately after it was removed from the sublimation chamber and the ice began to thaw.

\subsection{Low organic content}

We started the first series of measurements with an organicice-dust mass ratio of 5:100:300 and increased the dust content in two steps, to 5:100:500 and 5:100:700, respectively. In all measurements the reduction of the samples' volume was most noticeable as the ice sublimated (Fig. 2). No solid material detached in the form of ejected particles or cliff collapses, and we did not observe any transport away from the surface via other mass movement processes. As a result, none of the three samples produced visible areas where fresh material was exposed to the surface. In contrast to organic-free ice-dust mixtures, material loss from all organic-bearing samples appeared to be caused by sublimating ice only. On the lateral surface of the sample with the lowest dust content (5:100:300), the sample volume decreased and short fractures of $2-3 \mathrm{~mm}$ formed during the sublimation phase. This process slowed down during the experiment, and no further change was observed after $12 \mathrm{~h}$. With increasing dust content and a correspondingly lower amount of organic-rich ice, the formation of fractures became less evident. On the sample surface with the lowest content of the organic-rich ice $(5: 100: 700)$, no distinct fractures could be observed. On this sample, the surface alteration was generally less significant and stopped after $8 \mathrm{~h}$.

At the end of the experiment, the sample residues were removed from the sublimation chamber and a mantle of icedepleted material a few millimeters thick was observed. The mantle material was extremely porous and fragile but preserved its shape after the ice had sublimated. In contrast to the organicfree reference experiment, the ice-depleted material did not decay into granular dust under the influence of gravity. The icedepleted mantle covered the sample center, where ice remained preserved. As observed in the organic-free reference sample and in previous sublimation experiments (e.g., Poch et al. 2016b; Kaufmann \& Hagermann 2018; Haack et al. 2021), the icecontaining sample center was significantly solidified compared to the start of the experiment. No change in albedo before and after the experiment was detected on the side wall of any of the three samples.

\subsection{High organic content}

In the second series of measurements, the mass of organic material was doubled with respect to the amount of water ice. The mass ratios of dust were increased in three steps and were comparable to those of the first series of measurements (Table 2). The vertical insolation of 1.9 solar constants remained unchanged.

The samples of this measurement series presented a volume loss, which appeared qualitatively increased compared to samples with lower organic content (Fig. 3). No mass transport of solid material away from the surface was observed in any of the three samples. In the course of the experiments, distinct fractures developed on the lateral side of the samples. These fractures were more pronounced than in experiments with lower organic content, frequently exceeded $5 \mathrm{~mm}$ in length, and formed poorly developed networks. The angles at which they intersected were variable. The fractures evolved most distinctly, and the apparently highest volume loss was observed on the sample with the lowest dust content (10:100:300). The phase of observable surface alteration for this sample with the lowest dust content ended after about $12 \mathrm{~h}$.

In contrast to the first series, the sample with the highest dust content (10:100:700) also showed significant fracturing. However, the volume decrease was reduced and less rapid compared 

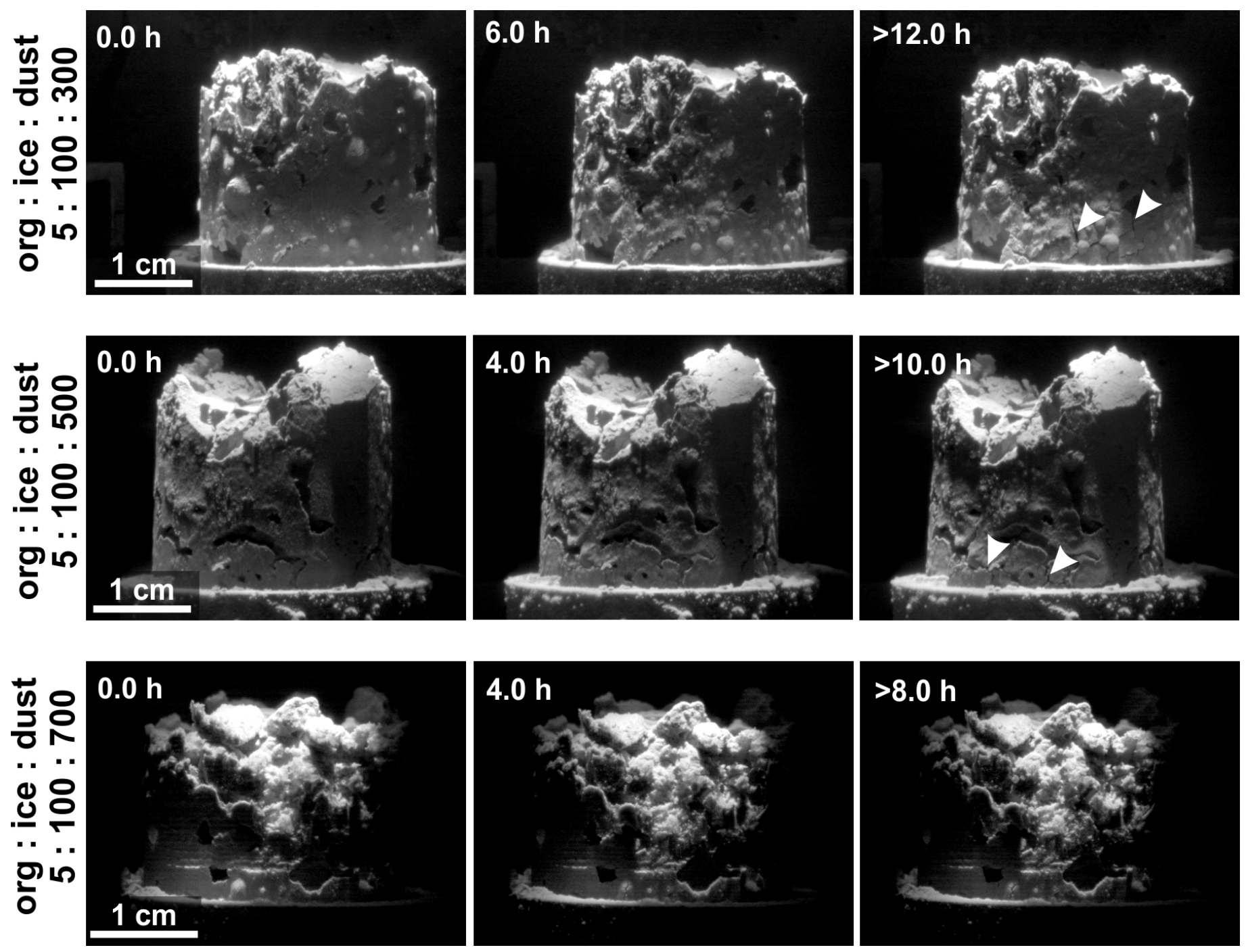

Fig. 2. Three vertically insolated samples with different organic-to-ice-to-dust mass ratios at an insolation rate of 1.9 solar constants. The image series show the alterations of the sample surfaces as a function of increasing dust content (online movies). The decreasing sample volume and formation of fractures (some marked with arrows) is most evident at a component mass ratio of 5:100:300 and reduces significantly with increasing dust content. The surface-altering activity of the first sample comes to an end after $12 \mathrm{~h}$. The following series show the dust-dependent reduction of the surface alterations and the timescales on which they occur. At a maximum relative dust amount of 5:100:700 per mass, the duration of visible sample alteration is reduced to $8 \mathrm{~h}$.

to samples with lower dust content. The phase of visible surface alteration of the sample with the highest dust content came to an end after about $6 \mathrm{~h}$. The comparison between the two series of measurements showed that the duration of the observable surface alteration depends predominantly on the dust content and not on the amount of the organic component. An increased dust content generally leads to reduced phases of surface alteration.

After the remains of the samples were removed from the sublimation chamber at the end of the experiments, an ice-depleted mantle on the surface, which enveloped an ice-rich core, was observed. This is in analogy to the results of the experiments with low organic content. The thickness of the mantle at the side surfaces reduced from more than 3 millimeters to about 1 millimeter as the dust content of the samples increased. Despite its high porosity, the mantle material was cohesive enough that fragments could be manually removed without disintegrating into granular dust. No change in the albedo of the sample materials before and after the experiments was observed.
The results of organic-rich sublimation experiments differ significantly from those without organics. The observed activities and morphologies at the sample surfaces are less diverse (no cliff collapses, outbursts etc.) and less dependent on the volatile content compared to the alterations of samples without organics (Fig. 1; see also Haack et al. 2021). In all observed samples, the surface alterations were dominated by volume loss and fracture formation that proceeded continuously with no distinct individual events.

\section{Discussion}

The observations obtained with the organic-free reference measurement are in line with those presented by Haack et al. (2021), even though the insolation flux is somewhat reduced compared to the previous experiments. The test shows that, with our experimental setup, a sample without organic components loses substantial amounts of nonvolatile material and is subject to 

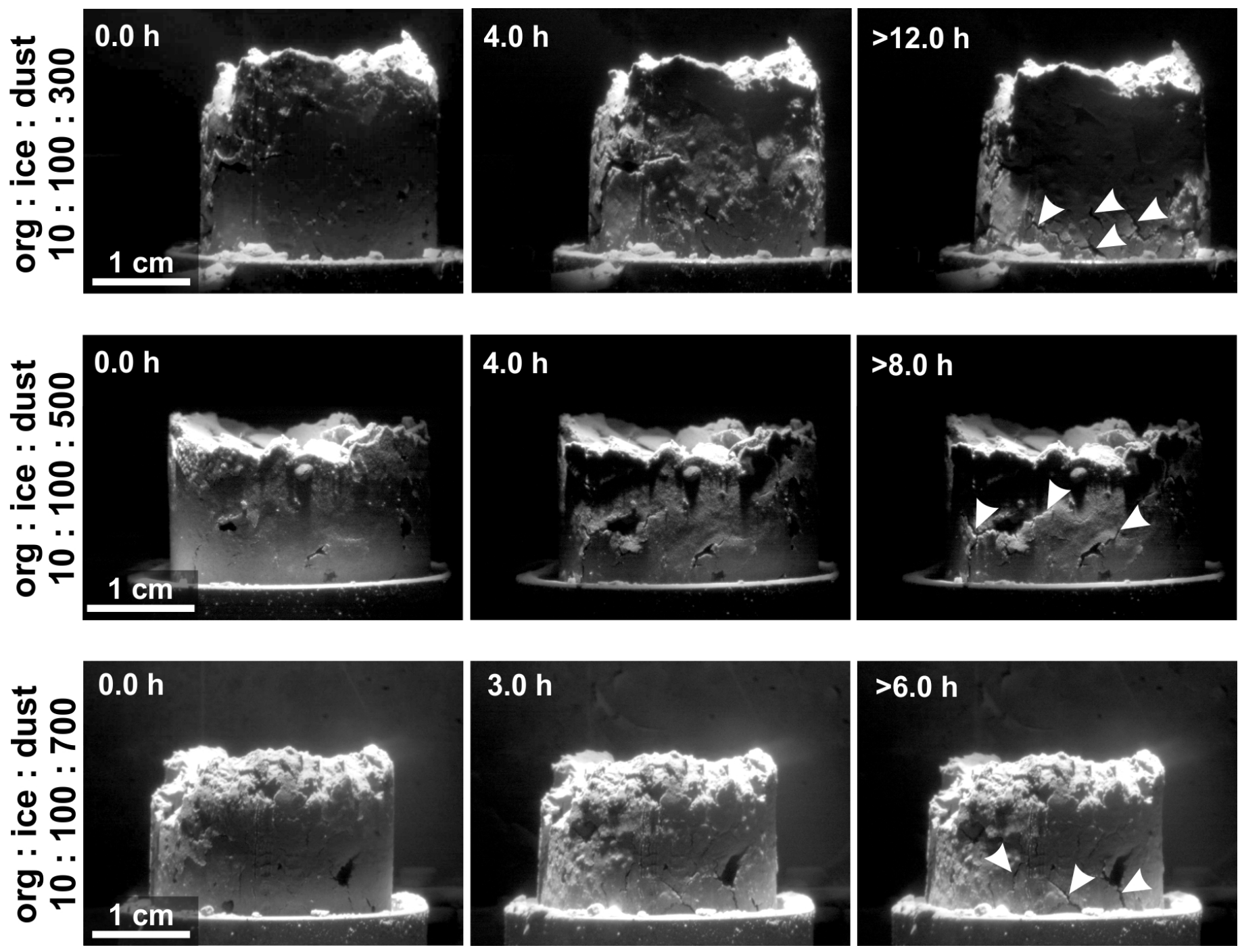

Fig. 3. Evolution of three sample surfaces with high organic content under vertical insolation. The reduction in the sample volume and the formation of fractures (some marked with arrows) can be observed in all three image series (online movies). This surface evolution is most distinctly observable in the top series, with an organic-to-ice-to-dust mass ratio of 10:100:300, whose phase of activity ended after $12 \mathrm{~h}$. The degree of surface alteration and its duration decreased with increasing amounts of the dust component and ended after 8 and $6 \mathrm{~h}$, respectively.

a significantly different morphological evolution than samples with organics.

At $150 \mathrm{~K}$, the addition of glycine and sodium acetate to water ice in a mass ratio of 10:100 only increases the surface energy of the ice particles within the measurement inaccuracy of the surface energy of pure water ice. We assume that this difference between organic-to-ice mixtures of 5:100 and pure ice is even smaller and cannot be determined with sufficient accuracy because of the limitations of the Brazilian disk test method. Therefore, we extrapolated the average surface energies of mixtures of dust, ice, and organics by using the values of previous Brazilian disk tests with ice-dust mixtures without organics (Haack et al. 2020) to estimate the tensile strength of each sample material.

The volume fractions of dust in the samples with different mixing ratios range from about $60 \mathrm{vol} \%$ for the lowest dust content (X:100:300) to almost 80 vol\% for the highest dust content (X:100:700; Table 2). The different amounts of organics have only a minor influence on the various volume fractions of the sample mixtures because of their small share in the total mass of the samples. With the sample's filling factor of 0.41
(Haack et al. 2021), the tensile strength values of the samples were calculated and range between $540 \mathrm{~Pa}$ for the lowest and 360 $\mathrm{Pa}$ for the highest dust content. This is somewhat above the high end predicted for materials on 67P by Groussin et al. (2015). Other estimates give an even lower strength of only a few pascals for cometary material (Attree et al. 2018a; O'Rourke et al. 2020). Since the size scaling is based on the comparison of cohesion on $67 \mathrm{P}$ and in the laboratory, it should be considered with caution. However, it is not unrealistic since hypothetical samples with a filling factor between 0.15 and 0.37 , as assumed for 67P (Fulle et al. 2016; Pätzold et al. 2016; Herique et al. 2019), would have a calculated tensile strength equivalent to that given by Groussin et al. (2015). With the method of sample preparation used, such low filling factors are not achievable. Therefore, the tensile strength in the laboratory is higher compared to on 67P.

\subsection{Low organic content}

In contrast to samples of previous sublimation experiments that investigated pure dust-ice mixtures, the samples with added organic components showed no broad diversity in their 
morphological evolution with respect to their variable volatile content. The two observed types of alterations were the reduction in the sample volume and the formation of fractures on the surface.

The volume reduction can be explained by the sublimation of the water ice. The organic components in the ice matrix (intramixture) remained in the sample after the sublimation of the ice and adhesively kept the dust particles in contact with one another. It is unlikely that significant amounts of organic material was transported away with the sublimating ice. Otherwise, analogous to the sample without organic components, the dust particles would have detached from the sample surface and fallen down (Fig. 1).

However, the effect of the organic material as a "glue" (Bischoff et al. 2020) holding the dust particles together remained sufficient to compensate for the loss of volatiles. Ice, with its higher surface energy compared to dust (Kimura et al. 2015; Gundlach et al. 2018b; Haack et al. 2020), prevents disintegration of the sample before its sublimation. After sublimation, a mixture of dust and organics remains, the tensile strength of which is higher than that of pure dust. Therefore, after the sublimation of the ice, the organic-rich samples do not disintegrate into individual dust particles, in contrast to what was observed in the reference measurement without organics and as described in Haack et al. (2021). The resulting free space was partially refilled by the remaining dust particles, which caused the samples to shrink. The observation that the ice-depleted material did not form a foam-like texture (Poch et al. 2016b) is probably due to the fact that the fraction of nonvolatile materials was much higher in our experiments. The reduced volume loss of samples with increased dust content can be explained by the lower ice content, which creates less free space after sublimation.

The reduced duration of sublimation activity results from the faster development of ice-depleted mantle material as the dust content of individual samples increases. The mantle protects underlying ice-containing layers from insolation and thus from further sublimation. A comparable process was already observed in previous experiments (e.g., Grün et al. 1993; Lämmerzahl et al. 1995; Haack et al. 2021), where an ice-depleted layer was formed of loose dust but not of cohesive material, unlike in our experiments.

The decrease in volume during sublimation created tensile stresses in the ice-depleted mantle and led to the formation of contraction cracks. This process is comparable to drying shrinkage due to the loss of volatiles and the formation of desiccation cracks on Earth (Peron et al. 2009; Sima et al. 2014). Desiccation is also one of the proposed processes responsible for the formation of fractures on comets (El-Maarry et al. 2015; Poulet et al. 2016). However, in the samples with a low content of organics, the fractures were only a few millimeters long, mostly isolated, and did not form networks. This could indicate that the amount of organic adhesive between the dust particles was sufficient to keep them attached to the original sample but allowed limited movement of the dust particles into the evolving free space. Accordingly, the ice-depleted material had a degree of plasticity that reduced the formation of fractures and prevented fractures altogether in the case of high dust contents. Another explanation for the absence of distinct fracture networks could be the limited surface area of the samples, which prevented the formation of extended fracture patterns.

Another process that can create fractures on the surface of a comet is repeated thermal contraction and expansion (El-Maarry et al. 2015; Spohn et al. 2015; Auger et al. 2018; Attree et al. $2018 b$ ). This is a slow process, requires periodical cycles of insolation, and produces polygonal patterns typical in periglacial landscapes on Earth (French 2017). In the laboratory, however, we did not perform insolation cycles and thus did not trigger any periodic thermal contraction or expansion. This explanation for the origin of fractures is therefore not applicable for our experiments. The formation of fractures by tectonics and compressive stress is conceivable on comets (Thomas et al. 2015b; Franceschi et al. 2020) but can be excluded in our laboratory experiments.

The formation of an ice-rich layer below the ice-depleted mantle can be explained by the recondensation and/or sintering of ice (Spohn \& Benkhoff 1990; Thomas et al. 1994; Seiferlin et al. 1995; Kossacki et al. 2015). This process has been observed many times in previous sublimation experiments (e.g., Grün et al. 1993; Poch et al. 2016b; Kaufmann \& Hagermann 2018) and is likely on 67P (Spohn et al. 2015; Lethuillier et al. 2016; Knapmeyer et al. 2018; Gundlach et al. 2020). In this process, water vapor migrates from near-surface layers deeper into the sample and recondenses there into a solid material (Kührt \& Keller 1994; Gundlach et al. 2020). Additionally, a temperature increase in near-surface layers due to insolated energy can trigger the sintering of ice particles (Gundlach et al. 2018a). In these cases, stiff connections of ice form between the particles and significantly increase the tensile strength of the material (Kuroiwa 1961; Gundlach et al. 2018a). Nevertheless, due to the reduced formation of fractures in this series of measurements, comparison with fracture-related morphologies of $67 \mathrm{P}$ is of limited significance.

\subsection{High organic content}

The doubled amount of organics in the second series of measurements enhanced the decrease in the sample volume and enhanced the formation of irregularly shaped fractures as the ice sublimated. The duration of the active phase of sample alteration was comparable to that of the first series with respect to the same ice-dust ratios. Due to the limitations of the experimental setup, the physical properties of the remaining material could not be measured after the experiment, so the analysis could only be done using the images taken by the camera (Fig. 3). Therefore, direct comparison of volume changes of different samples should be treated with caution since the whole sample was not observed and misperceptions could occur due to the camera's perspective. However, it is clear that the visually increased volume loss and intensified formation of fractures on the surface differ significantly compared to the first series. A possible explanation is increased inter-particle forces due to the higher organic content acting as an adhesive. The higher inter-particle forces would reduce the mobility of the particles and tend to make the mantle material less plastic compared with the low organics scenario (Valverde et al. 1998; Gröger et al. 2003). The consequence would be an increased formation of tensile fractures due to the volume loss. This process is comparable to the formation of desiccation cracks on Earth (Rayhani et al. 2008; Peron et al. 2009; Sima et al. 2014), which occur in a cohesive material due to the loss of volatiles.

As in the first series with low organic content, the possibility that the fractures result from thermal stress can be excluded. Such a possibility is contradicted by the fact that no periodically changing insolation was realized and that a large number of fractures occurred on the side surfaces of the samples, which were insolated only at very high angles of incidence.

Evolved networks of intersecting fractures are known to have been caused by terrestrial desiccation processes and on $67 \mathrm{P}$ may have been caused by thermal stresses (Spohn et al. 2015; 

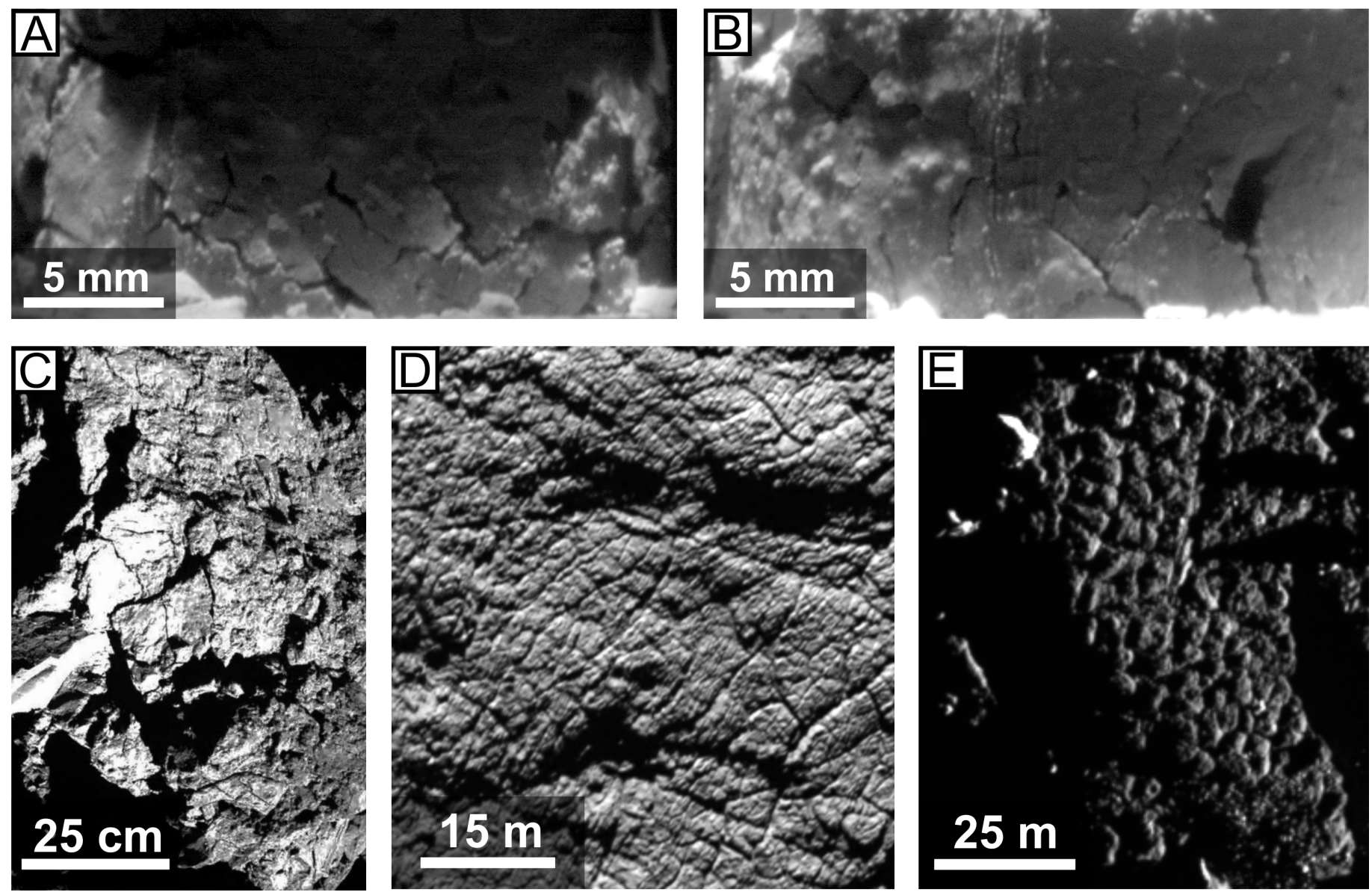

Fig. 4. Comparison of fractures generated in the laboratory (panels $A$ and $B$ ) and observed on 67P (panels $C-E$ ). Panel A: close-up of the sample with the lowest dust fraction (10:100:300) from Fig. 3. Distinct and irregularly shaped fractures form a poorly developed network on the side wall of the sample. Panel B: enlarged section of the sample with the highest dust content (10:100:700) from Fig. 3. The length and pattern of evolving fractures are comparable to those on samples with lower dust content, but they are significantly narrower. Panel C: detailed image of fractures on the surface of 67P, taken by the Philae lander. Several irregular cracks are visible on a rough and dust-free surface. The fracture patterns correspond most closely to those on panel B. Image credit: ESA/Rosetta/Philae/CIVA. Panel D: fracture network on the surface of 67P. The fractures form a pattern of polygons with widths of up to $5 \mathrm{~m}$. Panel E: evolved polygonal structures, caused by fractures on the surface of 67P. Image credits: ESA/Rosetta/MPS for OSIRIS Team MPS/UPD/LAM/IAA/SSO/INTA/UPM/DASP/IDA.

Auger et al. 2018; Attree et al. 2018b; El-Maarry et al. 2015, 2019), although none formed here. Occasionally, fractures intersected, but not at a preferred angle typical of polygonal fracture networks (Peron et al. 2009; Auger et al. 2018; French 2017). However, it cannot be excluded that the observed fractures represent an early stage of a slowly forming network or that polygonal networks could form in the analog material if the sample's surface area or the insolation rate were increased. These considerations suggest that polygonal networks do not necessarily have to be restricted to the near-surface hardened layer of a comet (Kochan et al. 1989; Kossacki et al. 2015; Spohn et al. 2015). Taking the size scaling of the laboratory experiments into account, irregular meter-sized desiccation cracks may form on a comet due to volatile loss (Poulet et al. 2016), while large polygonal networks could be generated by thermal stresses due to diurnal heating periods (e.g., Spohn et al. 2015; Auger et al. 2018; Attree et al. 2018b, see our Fig. 4).

As described in Sect. 4.2, larger and more numerous fractures were formed in volatile-rich samples. In parallel, the period of visible surface alteration and the thickness of the ice-depleted mantle are increased. This could indicate that fractures in volatile-rich samples propagate deeper into the material, where exposed volatiles also start to sublimate. This could cause existing fractures to propagate farther or new fractures to form. On a comet, these propagating fractures could locally increase the sublimation activity and lead to outgassing events (Skorov et al. 2016; Vincent et al. 2016; Höfner et al. 2017).

In all our experiments, water ice was the only volatile used. As mentioned in Sect. 2.1, water dominates the amount of all volatiles on 67P (e.g., Hässig et al. 2015; Läuter et al. 2018; Biver et al. 2019), and the potential sublimation and recondensation of $\mathrm{CO}_{2}$ does not result in a substantial solidification of a subsurface crust, since individual $\mathrm{CO}_{2}$ crystals do not form bridges to $\mathrm{H}_{2} \mathrm{O}$ crystals (Kochan et al. 1989). Therefore, water ice is expected to be the dominant volatile in the upper centimeters of the comet surface (Filacchione et al. 2016; Gundlach et al. 2020). Nevertheless, the influence of other volatiles on the evolution of a comet cannot be underestimated and remains a subject of investigation for future experiments.

The absence of collapse events or ejected fragments (Tubiana et al. 2015; Vincent et al. 2015a,b, 2016) from the sample surface could indicate that the sample mixtures do not simulate the actual composition of a comet accurately enough. It is also conceivable that an increased and periodically changing insolation in combination with the low albedo of a comet (Keller et al. 1986; Ciarniello et al. 2015) could have a significant effect on 
the evolution of the patterns and depths of fracture systems in future experiments.

\section{Conclusions}

A number of sublimation experiments were performed with samples containing variable amounts of organic, volatile, and dust components. The focus of the experiments was to study samples that contain the organic molecules glycine and sodium acetate (Elsila et al. 2009; Altwegg et al. 2016; Rubin et al. 2019; Schuhmann et al. 2019). Comparison with organic-free sample material showed that an insolation flux of 1.9 solar constants, equivalent to a simulated heliocentric distance of 3.6 $\mathrm{AU}$, was sufficient to sublimate the near-surface volatile water component and to alter the sample surface. The addition of a few mass percent of organic material to a volatile-rich sample fundamentally changes the evolution of the sample morphology. The evolution of sample surfaces with organics was characterized by a loss of volume and the formation of fractures at the surface. These fractures were compared with those on 67P (Spohn et al. 2015; El-Maarry et al. 2015; Poulet et al. 2016; Auger et al. 2018) and discussed with respect to their relevance to fracture formation on 67P.

The volatile content of the samples had a distinct influence on the duration of the surface alteration and on the degree of volume loss. The alteration of ice-rich samples lasted longer than that of samples with initially lower amounts of ice, and the former qualitatively lost more of their volume. These processes became less distinct with an increasing dust content of the samples. At the end of the experiments the thickness of ice-depleted material on the top and side surfaces was significantly higher for samples with initially high ice content compared to samples with lower ice content. This mantle prevented sublimation of ice from the sample center, and with increasing mantle thickness the surface alteration came to an end. The mantle material was extremely porous but was stable enough not to disintegrate into individual dust particles when mechanically stressed after the experiment. The nonvolatile organic components remained in the sample and acted as an adhesive between the individual dust particles, consolidating the ice-depleted material. The more organic material initially added to the sample, the more stable the ice-reduced mantle appeared.

In ice-depleted mantles with initially high organic content, the dust particles were less mobile compared to samples with low organic content. As a result, the mantle material became less plastic. The loss of sublimating ice caused the mantle material to contract, resulting in near-surface tensile stresses and the formation of fractures. These fractures propagated farther into the sample's subsurface as sublimation of the ice continued. This allowed further sublimation of ice from subsurface layers, which presumably would be protected from sublimation by a fracturefree mantle. The fractures in the experiment remained mostly separated from one another and did not form polygonal structures as observed on 67P (El-Maarry et al. 2015, 2016; Auger et al. 2018). However, this could be a result of the limited dimensions of the experimental setup. With a larger sample surface and a more intense insolation, the formation of polygonal structures due to volatile loss cannot be excluded. We conclude that the formation of fractures and, possibly, networks on a comet is not necessarily limited to a hardened subsurface layer and its diurnal thermal contraction (Spohn et al. 2015; Auger et al. 2018; Attree et al. 2018b). On meter scales, fractures on a comet could also result from the loss of sublimating volatiles, comparable to desiccation processes on Earth.
In our experiments we used nonvolatile-to-volatile ratios from 3 to 7 per mass, as inferred on 67P (Herique et al. 2017; Pätzold et al. 2018; Fulle et al. 2017, 2018) and confirmed in previous sublimation experiments (Haack et al. 2021). In future experiments, our restriction to glycine and acetate as organic analogs could be extended to other organic materials detected on 67P. Also, further experiments are necessary to investigate the influence of other volatiles, such as $\mathrm{CO}_{2}$, the influence of increased insolation flux, and the influence of a drastically reduced albedo on the morphological evolution of cometary surfaces.

Acknowledgements. This work was carried out in the framework of the CoPhyLab project funded by the D-A-CH programme (GU 1620/3-1 and BL 298/261/SNF 200021E 177964/FWF I 3730-N36). The research project "The Physics of Volatile-Related Morphologies on Asteroids and Comets" is funded by the DLR Management Board Young Research Group Leader Programme and the Executive Board Member for Space Research and Technology. We gratefully acknowledge their financial support and endorsement. Many thanks to I. Büttner from DLR Berlin for sieving the fly ash and to U. Altenberger from the University of Potsdam for providing SEM images and chemical analysis of fly ash.

\section{References}

Agarwal, J., A'Hearn, M. F., Vincent, J.-B., et al. 2016, MNRAS, 462, S78 Altwegg, K., Balsiger, H., Bar-Nun, A., et al. 2016, Sci. Adv., 2, e1600285 Altwegg, K., Balsiger, H., Berthelier, J., et al. 2017, MNRAS, 469, S130 Attree, N., Groussin, O., Jorda, L., et al. 2018a, A\&A, 611, A33 Attree, N., Groussin, O., Jorda, L., et al. 2018b, A\&A, 610, A76 Auger, A.-T., Groussin, O., Jorda, L., et al. 2018, Icarus, 301, 173 Bischoff, D., Gundlach, B., Neuhaus, M., \& Blum, J. 2019, MNRAS, 483, 1202 Bischoff, D., Kreuzig, C., Haack, D., Gundlach, B., \& Blum, J. 2020, MNRAS, 497, 2517

Biver, Bockelée-Morvan, D., Hofstadter, M., et al. 2019, A\&A, 630, A19

Blum, J., Schrapler, R., Davidsson, B. J. R., \& Trigo-Rodriguez, J. M. 2006, ApJ, $652,1768-81$

Blum, J., Gundlach, B., Mühle, S., \& Trigo-Rodriguez, J. M. 2014, Icarus, 235, 156

Blum, J., Gundlach, B., Krause, M., et al. 2017, MNRAS, 469, S755

Bockelée-Morvan, D., Crovisier, J., Erard, S., et al. 2016, MNRAS, 462, S170

Capaccioni, F., Coradini, A., Filacchione, G., et al. 2015, Science, 347, aaa0628

Chýlek, P., Ramaswamy, V., \& Srivastava, V. 1983, J. Geophys. Res.: Oceans, 88, 10837

Choukroun, M., Altwegg, K., Kührt, E., et al. 2020, Space Sci. Rev., 216, 44 Ciarniello, M., Capaccioni, F., Filacchione, G., et al. 2015, A\&A, 583, A31 Clark, R. N., \& Lucey, P. G. 1984, J. Geophys. Res.: Solid Earth, 89, 6341 Dodson-Robinson, S. E., Willacy, K., Bodenheimer, P., Turner, N. J., \& Beichman, C. A. 2009, Icarus, 200, 672

El-Maarry, M., Thomas, N., Gracia-Berná, A., et al. 2015, Geophys. Res. Lett., 42,5170

El-Maarry, M., Thomas, N., Gracia-Berná, A., et al. 2016, A\&A, 593, A110

El-Maarry, M. R., Groussin, O., Keller, H. U., et al. 2019, Space Sci. Rev., 215, 36

Elsila, J. E., Glavin, D. P., \& Dworkin, J. P. 2009, Meteoritics Planet. Sci., 44, 1323

Fanale, F. P., \& Salvail, J. R. 1984, Icarus, 60, 476

Filacchione, G., De Sanctis, M. C., Capaccioni, F., et al. 2016, Nature, 529, 368 Franceschi, M., Penasa, L., Massironi, M., et al. 2020, Proc. Natl. Acad. Sci. U.S.A., 117, 10181

French, H. M. 2017, The Periglacial Environment (John Wiley \& Sons) Fulle, M., Della Corte, V., Rotundi, A., et al. 2016, MNRAS, 462, S132 Fulle, M., Della Corte, V., Rotundi, A., et al. 2017, MNRAS, 469, S45 Fulle, M., Blum, J., Green, S. F., et al. 2018, MNRAS, 482, 3326 Goesmann, F., Rosenbauer, H., Bredehöft, J. H., et al. 2015, Science, 349 Gröger, T., Tüzün, U., \& Heyes, D. M. 2003, Powder Technol., 133, 203 Groussin, O., Jorda, L., Auger, A.-T., et al. 2015, A\&A, 583, A32

Grün, E., Bar-Nun, A., Benkhoff, J., et al. 1989, Int. Astron. Union Colloquium, 116,277

Grün, E., Gebhard, J., Bar?Nun, A., et al. 1993, J. Geophys. Res.: Planets, 98, 15091

Gulkis, S., Allen, M., von Allmen, P., et al. 2015, Science, 347, aaa0709

Gundlach, B., Blum, J., Keller, H. U., \& Skorov, Y. V. 2015, A\&A, 583, A12

Gundlach, B., Ratte, J., Blum, J., Oesert, J., \& Gorb, S. N. 2018a, MNRAS, 479 5272 
Gundlach, B., Schmidt, K. P., Kreuzig, C., et al. 2018b, MNRAS, 479, 1273 Gundlach, B., Fulle, M., \& Blum, J. 2020, MNRAS, 493, 3690

Haack, D., Otto, K. A., Gundlach, B., et al. 2020, A\&A, 642, A218

Haack, D., Lethuillier, A., Kreuzig, C., et al. 2021, A\&A, 649, A35

Hadraoui, K., Cottin, H., Ivanovski, S. L., et al. 2019, A\&A, 630, A32

Hässig, M., Altwegg, K., Balsiger, H., et al. 2015, Science, 347, aaa0276

Herique, A., Kofman, W., Beck, P., et al. 2017, MNRAS, 462, S516

Herique, A., Kofman, W., Zine, S., et al. 2019, A\&A, 630, A6

Höfner, S., Vincent, J.-B., Blum, J., et al. 2017, A\&A, 608, A121

Kappel, D., Sachse, M., Haack, D., \& Otto, K. A. 2020, A\&A, 641, A19

Kaufmann, E., \& Hagermann, A. 2018, Icarus, 311, 105

Keller, H. U., Arpigny, C., Barbieri, C., et al. 1986, Nature, 321, 320

Keller, H. U., Mottola, S., Hviid, S. F., et al. 2017, MNRAS, 469, S357

Kührt, E. 1984, Icarus, 60, 512-21

Kührt, E., \& Keller, H. U. 1994, Icarus, 109, 121

Kimura, H., Wada, K., Senshu, H., \& Kobayashi, H. 2015, ApJ, 812, 67

Knapmeyer, M., Fischer, H.-H., Knollenberg, J., et al. 2018, Icarus, 310, 165

Kochan, H., Roessler, K., Ratke, L., et al. 1989, in ESA SP 302, Physics and Mechanics of Cometary Materials, eds. J. J. Hunt \& T. D. Guyenne, 115

Kossacki, K., Kömle, N., Leliwa-Kopystyński, J., \& Kargl, G. 1997, Icarus, 128, 127

Kossacki, K. J., Spohn, T., Hagermann, A., Kaufmann, E., \& Kührt, E. 2015, Icarus, 260, 464

Kuroiwa, D. 1961, Tellus, 13, 252

Lämmerzahl, P., Gebhard, J., Grün, E., \& Klees, G. 1995, Planet. Space Sci., 43, 363-73

Läuter, M., Kramer, T., Rubin, M., \& Altwegg, K. 2018, MNRAS, 483, 852

Lethuillier, A., Le Gall, A., Hamelin, M., et al. 2016, A\&A, 591, A32

Lorek, S., Gundlach, B., Lacerda, P., \& Blum, J. 2016, A\&A, 587, A128

Lorek, S., Lacerda, P., \& Blum, J. 2018, A\&A, 611, A18

Musiolik, G., \& Wurm, G. 2019, ApJ, 873, 58

Oehler, A., \& Neukum, G. 1991, Geophys. Res. Lett., 18, 253

O’Rourke, L., Heinisch, P., Blum, J., et al. 2020, Nature, 586, 697
Pajola, M., Oklay, N., Forgia, F. L., et al. 2016, A\&A, 592, A69 Pätzold, M., Andert, T., Hahn, M., et al. 2016, Nature, 530, 63

Pätzold, M., Andert, T. P., Hahn, M., et al. 2018, MNRAS, 483, 2337

Peron, H., Laloui, L., Hueckel, T., \& Bo Hu, L. 2009, Eur. J. Environ. Civil Eng., 13,869

Poch, O., Pommerol, A., Jost, B., et al. 2016a, Icarus, 266, 288

Poch, O., Pommerol, A., Jost, B., et al. 2016b, Icarus, 267, 154

Pommerol, A., Jost, B., Poch, O., et al. 2015, Planet. Space Sci., 109-110, 106

Poulet, F., Lucchetti, A., Bibring, J.-P., et al. 2016, MNRAS, 462, S23

Raponi, A., Ciarniello, M., Capaccioni, F., et al. 2020, Nat. Astron., 5, 500

Rayhani, M., Yanful, E., \& Fakher, A. 2008, Eng. Geol., 97, 25

Rubin, M., Altwegg, K., Balsiger, H., et al. 2019, MNRAS, 489, 594

Schuhmann, M., Altwegg, K., Balsiger, H., et al. 2019, ACS Earth Space Chem., 3,1854

Seiferlin, K., Spohn, T., \& Benkhoff, J. 1995, Adv. Space Res., 15, 35

Sierks, H., Barbieri, C., Lamy, P. L., et al. 2015, Science, 347, aaa1044

Sima, J., Jiang, M., \& Zhou, C. 2014, Comput. Geotech., 56, 168

Skorov, Y., Rezac, L., Hartogh, P., Bazilevsky, A., \& Keller, H. 2016, A\&A, 593, A76

Snodgrass, C., Tubiana, C., Bramich, D. M., et al. 2013, A\&A, 557, A33

Spohn, T., \& Benkhoff, J. 1990, Icarus, 87, 358

Spohn, T., Knollenberg, J., Ball, A. J., et al. 2015, Science, 349

Thomas, H., Ratke, L., \& Kochan, H. 1994, Adv. Space Res., 14, 207

Thomas, N., Davidsson, B., El-Maarry, M. R., et al. 2015a, A\&A, 583, A17

Thomas, N., Sierks, H., Barbieri, C., et al. 2015b, Science, 347, aaa0440

Tubiana, C., Snodgrass, C., Bertini, I., et al. 2015, A\&A, 573, A62

Valverde, J. M., Ramos, A., Castellanos, A., \& Keith Watson, P. 1998, Powder Technol., 97, 237

Vincent, J.-B., Bodewits, D., Besse, S., et al. 2015a, Nature, 523, 63

Vincent, J.-B., Oklay, N., Marchi, S., Höfner, S., \& Sierks, H. 2015b, Planet. Space Sci., 107, 53

Vincent, J.-B., Oklay, N., Pajola, M., et al. 2016, A\&A, 587, A14

Weissman, P., Morbidelli, A., Davidsson, B., \& Blum, J. 2020, Space Sci. Rev., 216,1 